\title{
User Association in 5G Heterogeneous Networks Exploiting Multi-Slope Path Loss Model
}

Conference Paper · February 2017

DOI: 10.1109/RTTR.2017.7887875

CITATIONS

0

5 authors, including:

\section{Syed Ali Hassan}

National University of Sciences \& Technology

94 PUBLICATIONS 370 CITATIONS

SEE PROFILE

\section{Qiang Ni}

Lancaster University

156 PUBLICATIONS 3,321 CITATIONS

SEE PROFILE
READS

46

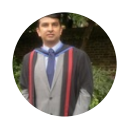

Haris Pervaiz

Lancaster University

21 PUBLICATIONS 99 CITATIONS

SEE PROFILE

\section{Leila Musavian}

University of Essex

63 PUBLICATIONS 1,258 CITATIONS

SEE PROFILE

Some of the authors of this publication are also working on these related projects: 


\title{
User Association in 5G Heterogeneous Networks Exploiting Multi-Slope Path Loss Model
}

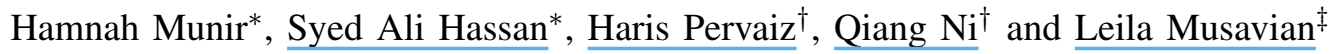 \\ *School of Electrical Engineering \& Computer Science (SEECS), National University of Sciences \& Technology \\ (NUST), Islamabad, Pakistan \{14mseehmunir, ali.hassan $\} @$ seecs.edu.pk \\ ${ }^{\dagger}$ School of Computing \& Communications, Lancaster University, UK. \\ $\{$ h.pervaiz, q.ni\}@lancaster.ac.uk \\ ${ }^{\ddagger}$ School of Computer Science \& Electronic Engineering, Essex University, UK. \\ $\{$ Leila.Musavian\}@essex.ac.uk
}

\begin{abstract}
Traffic offloading via small cells is important to realize the benefits of multi-tier heterogeneous networks (HetNets). Currently, the user association techniques are under the influence of single slope path loss model. The densification of networks and irregular cell patterns have increased the variations in both the link distances and interferences; making single slope path loss models less accurate. In this paper, we consider the downlink of a HetNet with picocells overlaid on a macrocell and propose a framework for user association with dual slope path loss model. Simulation results show that the dual slope model improves the system performance compared to the standard single slope model by offloading more traffic from macro-tier to pico-tier; the effect being more significant at higher edge user density. Furthermore, the user association is highly dependent on the path loss exponents in a dual slope model.
\end{abstract}

Index Terms-Heterogeneous network, dual slope path loss model, user association, load balancing.

\section{INTRODUCTION}

Heterogeneous networks (HetNets) have emerged as an effective solution to overcome the challenges triggered due to drastic growth of wireless data traffic. They ensure significant enhancement in the overall network performance that include high data rates and expanded cell coverage [1]-[3]. Nevertheless, these perks are accompanied by new technical challenges namely hardware expenses, interference management, user association, load balancing, radio resource management, energy efficiency along with the others [4].

Recently, numerous studies have focused on the mixed deployment using macrocell and distributed small cells, which have shown significant results to get higher throughput gains in dense networks. To manage the high user density and to increase the capacity, it is desirable to shift the traffic load from macrocell to small cells. HetNets, consisting of small cells with smaller coverage range, allow small cell base stations (BSs) to communicate at lower powers which limits the fraction of users connected to them, resulting in congestion at the macro-tier. Different load balancing techniques are studied to offload the traffic from macro-tier [5], [6]. One promising way to resolve this issue is through static cell biasing that allows users to offload to small cells using a biased measured signal. This suboptimum offloading technique is known as cell range expansion. However, the traffic demand in hot spots in the dense networks often varies with time, which calls to dynamically adjust the biases, resulting in enhanced load balancing gains [7], [8].

Most of the existing literature uses single slope path loss model to represent the path loss over the entire coverage range. While this model is easy to study and analyze, it does not capture the dependence of path loss exponent on the link distance perfectly. However, in the most recent works, this trend is shifted more towards dual slope path loss model. This migration is influenced by the network densification and recent work on millimeter wave (mmWave) communications because of highly intermittent links [9]. Multi-slope models apply different slopes for different link distances, which result in improved performance for dense networks [10]. In [11], dual slope path loss models are used to study the coverage probability with varying user density. The authors in [12] extended this work to user association in HetNets using dual slope path loss model.

In this paper, we extend the dual slope analysis on the downlink of a HetNet with picocells overlaid on a macrocell. The user association is done to offload the traffic to picotier using dual slope path loss model. We have considered different slopes before and beyond the critical distance, which can be used to approximate the two regimes of line of sight (LOS) and non line of sight (NLOS) links. This distance is environment dependent, which increases with less blocking environment, but can be approximated by taking the average LOS link distance. The performance enhancement with dual slope model is significant in achieving better offloading compared to single slope model in HetNets. The user association and load balancing is analyzed and we show that the biasing with dual slope path loss model outperforms the conventional biasing schemes. The dual slope path loss model offloads the users to the nearby small cells, thus offloading the traffic from macro-tier.

The rest of this paper is organized as follows. In Section II, we present the system model of the proposed framework along with path loss models. Section III shows the simulation results to demonstrate the performance of proposed scheme under single and dual slope path loss models and Section IV concludes the paper. 


\section{SYSTEM MODEL}

Consider the downlink of a two-tier HetNet composed of $M-1$ picocell base station (PBSs) overlaid on a macrocell. A snapshot of a two-tier HetNet is shown in Fig. 1 where both tiers use dual-slope path loss model. The path loss models are explained in detail in Section II-A. The macrocell base station (MBS) is represented by $m_{o}$ whereas the set of all the base stations (BSs) in the system is given as $\mathbb{M}=\left\{m_{o}, m_{1}, \ldots, m_{M-1}\right\}$. Let $\mathbb{N}=\mathbb{N}_{M} \cup \mathbb{N}_{o}$ be the set of all users deployed randomly over the entire area. The set of macrocell user equipments (MUEs) is denoted by $\mathbb{N}_{\odot}$ and the set of picocell users equipments (PUEs) is represented by $\mathbb{N}_{M}=\bigcup_{m=1}^{M-1} \mathbb{N}_{m}$ where $\mathbb{N}_{m}$ is the set of PUEs served by the $m^{\text {th }}$ PBS.

\section{A. Path Loss Models}

In this section, we present different path loss models to model the large scale fading in the network. The single slope path loss model is given as

$$
L(d)[\mathrm{dB}]=20 \log _{10}\left(\frac{4 \pi}{\lambda_{c}}\right)+10 \alpha \log _{10}(d)+\xi,
$$

where $\lambda_{c}$ corresponds to the carrier wavelength, $\alpha$ is the path loss exponent and $\xi$ is a Gaussian random variable (RV) with zero mean and $\sigma^{2}$ variance.

The single slope path loss model is the standard model, which falls short in accurately capturing the path loss exponent dependence on the physical environment in dense and millimeter wave capable networks. These limitations lead to the consideration of dual-slope path loss model in future networks.

The dual-slope path loss model is given as

$$
L(d)[\mathrm{dB}]=\left\{\begin{array}{ll}
\beta+10 \alpha_{1} \log _{10}(d)+\xi & d \leq r_{c} \\
\beta+10 \alpha_{1} \log _{10}\left(r_{c}\right) & \\
+10 \alpha_{2} \log \left(\frac{d}{r_{c}}\right)+\xi & d>r_{c}
\end{array},\right.
$$

where $d$ is the distance in meters and $r_{c}$ is the critical distance. $\beta$ represents the floating intercept, $\alpha_{1}$ and $\alpha_{2}$ are the slopes for below and beyond critical radius, $r_{c}$.

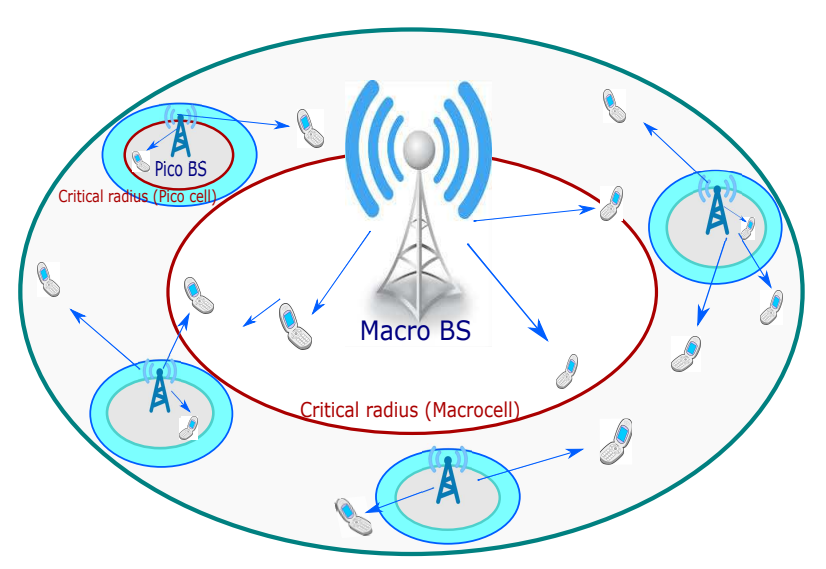

Figure 1. A two-tier heterogeneous network with red circles showing the critical radius of picocell and macrocell.
Table I

PARAMETER NOTATION.

\begin{tabular}{|c|c|}
\hline Parameter & Symbols \\
\hline Set of Tiers & $\mathbb{~}$ \\
\hline Set of BSs & $\mathbb{M}$ \\
\hline Set of Users & $\mathbb{N}$ \\
\hline Transmit Power & $p_{n, m}$ \\
\hline Channel Gain & $h_{n, m}$ \\
\hline Channel-to-interference-plus-noise Ratio & $\gamma_{n, m}$ \\
\hline$i^{t h}$ tier Biasing Factor & $\theta_{i}$ \\
\hline Critical Radius & $r_{c}$ \\
\hline Path Loss Exponent (Single-Slope & $\alpha$ \\
\hline Model) & \\
\hline Path Loss Exponents (Dual-Slope \\
Model) & {$\left[\alpha_{1}, \alpha_{2}\right]$} \\
\hline Floating intercept (Dual Slope) & $\beta$ \\
\hline$m^{t h}$ BS Power Budget & $P_{m}^{\max }$ \\
\hline Noise Power & $N_{0}$ \\
\hline
\end{tabular}

This dual slope model can be generalized into N-slope model as

$$
L(d)[\mathrm{dB}]=\left\{\begin{array}{lc}
l_{1}(d)=\beta+10 \alpha_{1} \log _{10}(d)+\xi & 0<d \leq r_{c}^{(1)} \\
l_{2}\left(r_{c}^{(1)}, d\right)=l_{1}\left(r_{c}^{(1)}\right)+ & r_{c}^{(1)}<d \leq r_{c}^{(2)} \\
10 \alpha_{2} \log \left(\frac{d}{r_{c}^{(1)}}\right) & \\
l_{3}\left(r_{c}^{(1)}, r_{c}^{(2)}, d\right)=l_{2}\left(r_{c}^{(1)}, r_{c}^{(2)}\right) & \\
+10 \alpha_{3} \log \left(\frac{d}{r_{c}^{(2)}}\right) & r_{c}^{(2)}<d \leq r_{c}^{(3)} \\
\cdot & \\
\cdot & \\
\cdot & \\
l_{N}\left(r_{c}^{(1)}, r_{c}^{(2)}, \ldots, r_{c}^{(N-1)}, d\right)= & \\
l_{N-1}\left(r_{c}^{(1)}, r_{c}^{(2)}, \ldots, r_{c}^{(N-1)}\right)+ & \\
10 \alpha_{N} \log \left(\frac{d}{r_{c}^{(N-1)}}\right) & d>r_{c}^{(N-1)}
\end{array}\right.
$$

where $\alpha_{n}, n=\{1, \ldots, N\}$, is the path loss exponent such that $0 \leq \alpha_{1} \leq \alpha_{2} \leq \ldots \leq \alpha_{N}$. The critical distance is denoted as $r_{c}^{(n)}, n=\{1, . ., N-1\}$, such that $r_{c}^{(1)} \leq r_{c}^{(2)} \leq \ldots \leq r_{c}^{(N)}$.

\section{B. User Association}

This paper considers different approaches for user association. We assume open access, which allows users to connect to any tier. We analyze the cell association based on minimum path loss, maximum biased received power and maximum biased rate.

1) Minimum Path Loss: We first consider the association on the basis of path loss, where users are associated with the $\mathrm{BS}$ which gives the lowest path loss. The $n^{\text {th }}$ user is associated with the $m^{\text {th }}$ BS that maximizes

$$
\underset{m}{\arg \max } \frac{1}{L\left(d_{n, m}\right)},
$$

where $d_{n, m}$ is the distance between the $n^{t h}$ user and the $m^{t h}$ BS. 
2) Maximum Biased Received Power: The association is determined on the basis of received power, where users are associated with the BS that serves the maximum biased received power. The $n^{t h}$ user is associated with the $m^{t h}$ BS that maximizes

$$
\underset{m}{\arg \max } \frac{\theta_{i} P_{m}^{\max }}{L\left(d_{n, m}\right)},
$$

where $P_{m}^{\max }$ is the maximum transmit power of the $m^{\text {th }}$ BS and $\theta_{i}$ is the bias factor for the $i^{t h}$ tier and all the BSs in the particular tier use the identical bias value. This case can be reduced to maximum received power association by putting $\theta_{i}=1$. This paper assumes the bias value for macro-tier, $\theta_{1}=0 \mathrm{~dB}$ and it varies between $0 \mathrm{~dB}$ to $30 \mathrm{~dB}$ for pico-tier, in case of biased received power association.

3) Maximum Biased Rate: The user association is decided on the basis of achievable rate. The $n^{\text {th }}$ user is associated with the $m^{t h}$ BS that gives the maximum biased rate, i.e,

$$
\underset{m}{\arg \max } \theta_{i} R_{n, m},
$$

where $\theta_{i}$ is the bias factor for the $i^{t h}$ tier. This paper assumes the bias value for macro-tier, $\theta_{1}=1$, in case of biased rate association. The achievable rate, $R_{n, m}$, in $(\mathrm{b} / \mathrm{s} / \mathrm{Hz})$ can be formally defined as

$$
R_{n, m}=\log _{2}\left(1+p_{n, m} \gamma_{n, m}\right),
$$

where $p_{n, m}$ is the transmit power from the $m^{t h} \mathrm{BS}$ to the $n^{\text {th }}$ user. $\gamma_{n, m}$ is the channel-to-noise ratio between the $m^{\text {th }}$ BS and the $n^{\text {th }}$ user.

The channel-to-noise ratio is defined as

$$
\gamma_{n, m}=\frac{\left|h_{n, m}\right|^{2}}{N_{0}}
$$

where $N_{0}$ represents the noise power and $h_{n, m}$ corresponds to the channel gain. In this paper, we assume that each user is associated with one BS at a time.

\section{Simulation Results}

We consider a two-tier HetNet with a single macrocell of radius $500 \mathrm{~m}$ where picocells are uniformly overlaid on the edge of it. The maximum transmit power of MBS and PBS, $P_{m}^{\max }$, is set to $46 \mathrm{dBm}$ and $30 \mathrm{dBm}$, respectively. The power spectral density of noise is $-174 \mathrm{dBm} / \mathrm{Hz}$. The parameters used for path loss models are listed in Table II [13], unless stated otherwise.

For user deployment, two different schemes are considered. In the first scheme, users are uniformly scattered over the entire area whereas, in the second scheme, high user density exists outside the critical radius of the macrocell.

Fig. 2 and Fig. 3 show the fraction of users associated with pico-tier for maximum biased received power association and uniform user deployment. The values of path loss exponents used in these figures represent harsh and moderate environment conditions, respectively.

In Fig. 2, biasing effect is investigated by varying the bias factor of the pico-tier with no biasing of the macro-
Table II

SiMULATION PARAMETERS.

\begin{tabular}{|c|c|c|c|}
\hline Parameter & Value & Parameter & Value \\
\hline$\beta$ & $42.1 \mathrm{~dB}$ & $\sigma_{\xi}$ & $6.9 \mathrm{~dB}$ \\
\hline$\alpha$ & 3 & $f_{c}$ & $2.4 \mathrm{GHz}$ \\
\hline$r_{m}$ (macrocell $)$ & $350 \mathrm{~m}$ & $r_{m}($ picocell $)$ & $50 \mathrm{~m}$ \\
\hline
\end{tabular}

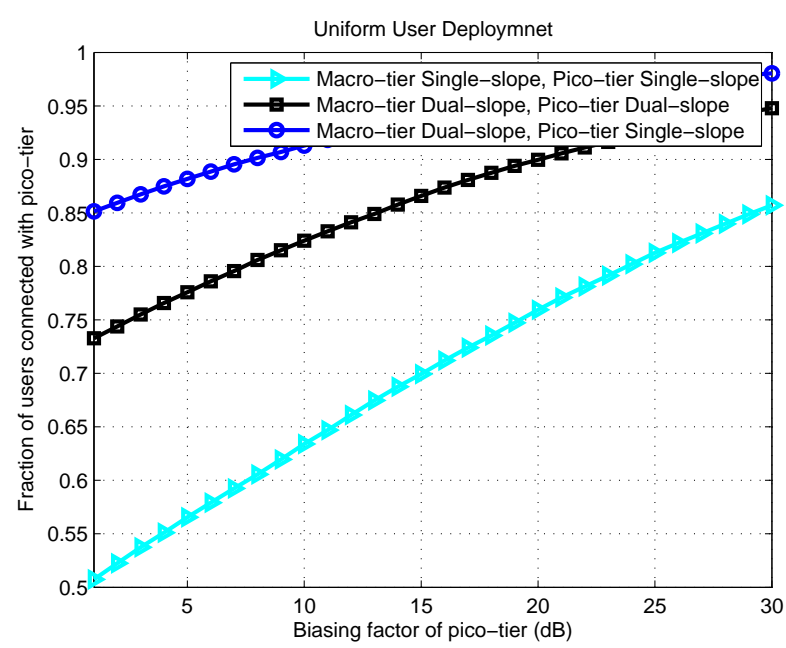

Figure 2. Fraction of users connected to pico-tier when biased received power association is used across varying biasing factor of pico-tier, $\theta_{2}$, for $N=100$, $M=4, \theta_{1}=0 \mathrm{~dB},\left[\alpha_{1}, \alpha_{2}\right]($ Macro-tier $)=[4,5]$ and $\left[\alpha_{1}, \alpha_{2}\right]($ Pico-tier $)=$ $[3,4]$.

tier for harsh environment conditions. An increasing trend in user offloading can be observed with the increasing pico-tier bias factor as biasing improves the received signal strength originating from PBSs. The figure reveals that biasing with both single and dual slope models is beneficial for offloading. However, with dual-slope model, this effect is stronger as dual slope model better approximates the links. This figure also compares the offloading performance of the network while exploiting single-slope and dual-slope path loss models. The figure shows that the offloading is maximum with dual slope model in the macrocell, as higher path loss exponents of the macro-tier directs the users to the nearby BSs due to highly attenuated long distance links between users and MBS. As the user leaves the critical radius of the macrocell, the NLoS path loss exponent increases, which further decreases the signal strength and users are offloaded to pico-tier. In harsh environment conditions, applying dual slope model in the macrocell offloads the traffic to pico-tier and if dual slope model is applied on picocells too, it prevents the offloading up to some extent as NLoS exponent of pico-tier is greater than the PLE used for single slope model.

Fig. 3 shows the fraction of users associated with picotier for moderate environment conditions and rest of the assumptions are same as used in Fig. 2. This figure reveals that the performance of the scheme with dual slope model in both tiers is better than the scheme where dual slope model is applied on macro-tier only, unlike the previous case. This is because of the fact that in moderate environment conditions, 


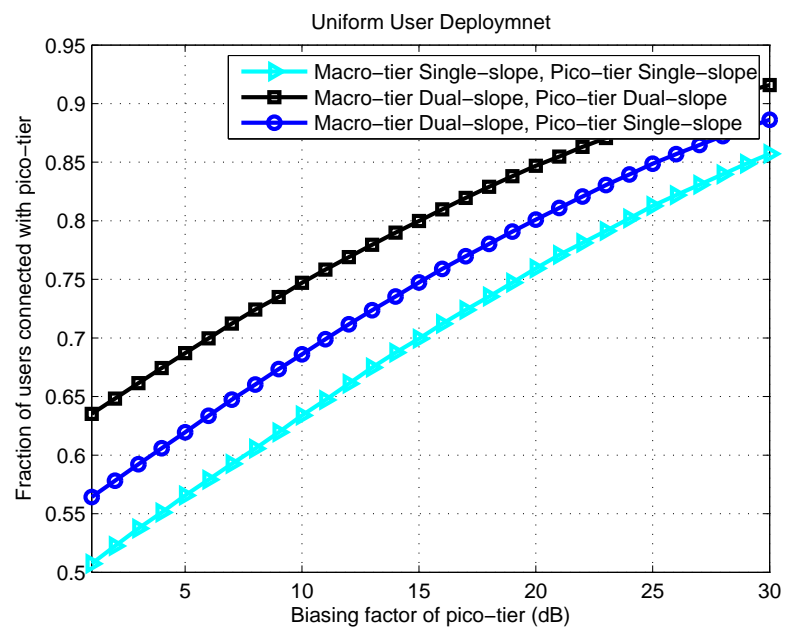

Figure 3. Fraction of users connected to pico-tier when biased received power association is used across varying biasing factor of pico-tier, $\theta_{2}$, for $N=100$, $M=4, \theta_{1}=0 \mathrm{~dB},\left[\alpha_{1}, \alpha_{2}\right]$ (Macro-tier $)=[3,4]$ and $\left[\alpha_{1}, \alpha_{2}\right]($ Pico-tier $)=$ $[2,4]$.

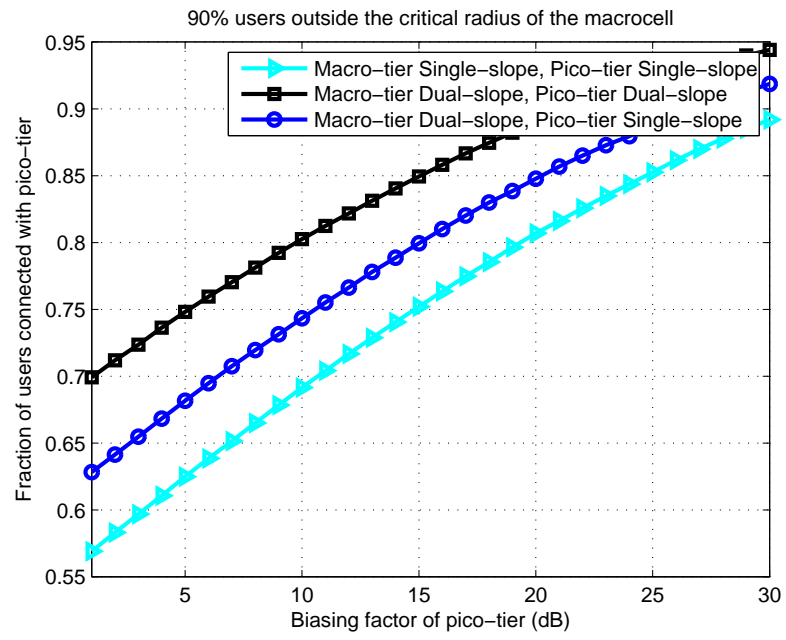

Figure 4. Fraction of users connected to pico-tier when biased received power association is used across varying biasing factor of pico-tier, $\theta_{2}$, for $N=100$, $M=4, \theta_{1}=0 \mathrm{~dB},\left[\alpha_{1}, \alpha_{2}\right]$ (Macro-tier) $=[3,4]$ and $\left[\alpha_{1}, \alpha_{2}\right]$ (Pico-tier) $=$ $[2,4]$.

lower path loss exponent within the critical radius induces less attenuation. The offloading to pico-tier is comparatively less when dual slope model is used in macro-tier only as some users residing within the critical radius of the macrocell might prefer MBS over PBSs because smaller PLE is used within the critical radius of the macrocell, resulting in reduced attenuation. The offloading improves when dual slope model is applied on pico-tier too, as more users are pushed toward nearby PBSs with less attenuated coverage region.

Fig. 4 shows the fraction of users associated with pico-tier across varying biasing factor of pico-tier. Maximum received power association and high edge user density is considered with moderate environment conditions. The figure shows that the offloading is relatively high in this case as compared to the previous case where uniform user deployment is used as

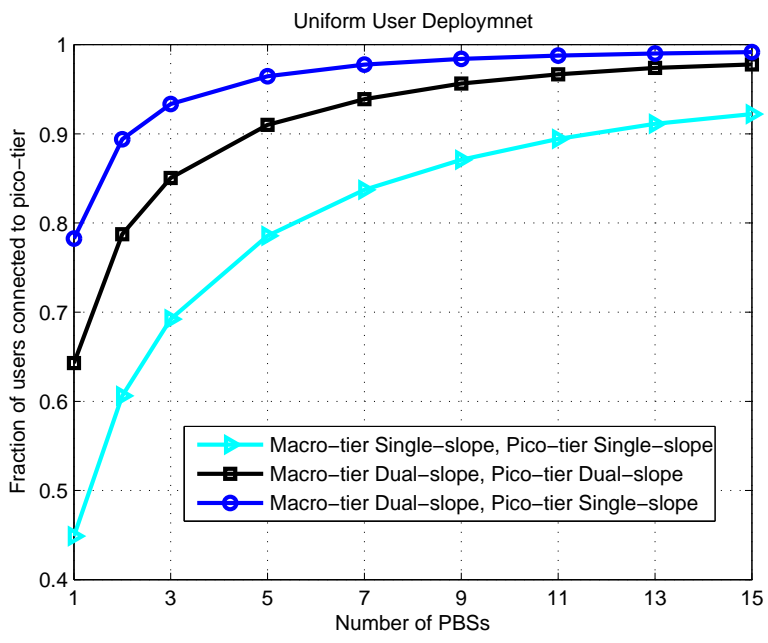

Figure 5. Fraction of users connected to pico-tier when path loss association is used across varying density of PBSs for $N=100, \theta_{1}=\theta_{2}=0 \mathrm{~dB}$, $\left[\alpha_{1}, \alpha_{2}\right]$ (Macro-tier) $=[4,5]$ and $\left[\alpha_{1}, \alpha_{2}\right]$ (Pico-tier) $=[3,4]$.

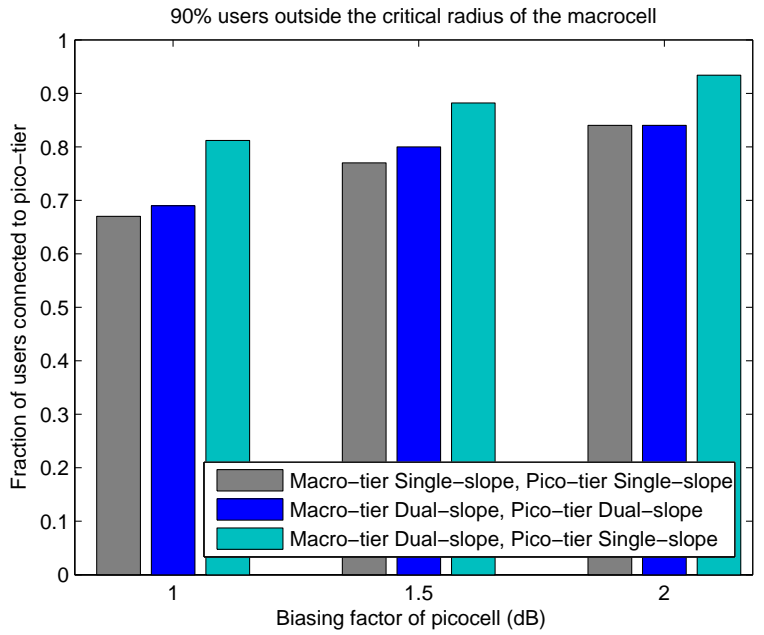

Figure 6. Fraction of users connected to pico-tier when association is done based on biased maximum rate across varying pico-tier bias factor, $\theta_{2}$, for $N=50, M=4, \theta_{1}=0 \mathrm{~dB},\left[\alpha_{1}, \alpha_{2}\right]$ (Macro-tier) $=[4,5]$ and $\left[\alpha_{1}, \alpha_{2}\right]$ (Pico-tier) $=[3,4]$.

shown in fig. 3. This is due to the fact that the picocells are deployed on the edge of the macrocell where the density of users is high for this case and thus, the offloading improves. This figure further reveals that the dual slope model needs less biasing to achieve a particular offloading as compared to single slope model.

In Fig. 5, the path loss association is considered to show the impact of BS density on the user offloading to pico-tier. The figure shows that as the density of PBSs increases, the distances of the users from the PBSs decreases, which in turn decreases the path losses and the load is shifted to the less congested PBSs. The trend is sharp in the start as the edge users start connecting to the pico-tier, which is more rapid in case of dual slope model. This offloading almost becomes invariant with further increase in the PBSs density in case of dual slope model. 


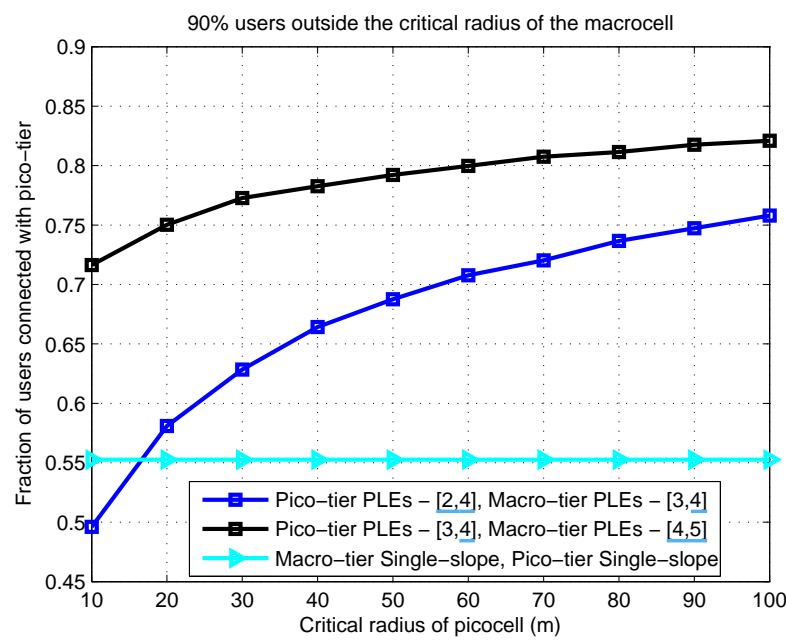

Figure 7. Fraction of users connected to pico-tier when biased received power association is used across varying critical radius of picocell for $N=100$, $M=4, \theta_{1}=\theta_{2}=0 \mathrm{~dB}$

Fig. 6 shows the user association in case of rate maximization for high edge user density. Similar trend as in Fig. 2 can be seen here. The fraction of the users associated to picotier increases with the dual slope model but the improvement in offloading, is comparatively less when compared to other two association schemes. This is because of the fact that the dual slope model is more beneficial for median users as compared to the edge users in terms of high data rates. Thus, less number of users offload to pico-tier in order to maximize their rate, however, the offloading is better as compared to the single slope model. This figure also reveals that the increase in the bias factor for pico-tier improves the offloading, as users get better biased rate from the pico-tier.

In fig. 7, we demonstrate the impact of critical radius of the picocell on the performance of the network for high edge user deployment. As the critical radius of the picocell increases, more users start entering within $r_{c}$, the attenuation decreases due to smaller PLE and the users residing within $r_{c}$ prefer PBSs due to less attenuated links. However, the increasing trend in the sum rate is sharp in the beginning and then it starts slowing down with further increase in $r_{c}$. This is because of the fact that as $r_{c}$ increases, the user offloading to pico-tier increases but the distance between the PBSs and the users also increases and the approximation of LoS links within the critical radius of picocells start affecting. The figure further reveals the impact of path loss exponents of the dual slope model on user offloading. It can be seen from the figure that the case with larger path loss exponents shows better offloading as they induce higher attenuation in the cell and users prefer nearby BSs. The user offloading in case of single slope model is minimum as it does not accurately characterize the network, which cause performance degradation.

\section{CONCLUSION}

In this paper, we analyzed the impacts of dual slope path loss model on the performance of a downlink multi-tier HetNet where different path loss exponents are used for different ranges. The user association is done to offload the traffic from macro-tier to pico-tier under single and dual slope path loss models. Simulation results showed that the dual slope model shows significant improvement in terms of load balancing in comparison to single slope model, which does not measure the path loss exponent dependence on the link distance accurately. With the dual slope model, more users offload to pico-tier with lower biasing as compared to single slope model. We also proved that the user association is highly dependent on the path loss exponents of the dual slope model. The above results strengthen the position of multi slope path loss model as a potential substitute for standard path loss model in the ever denser future networks.

\section{REFERENCES}

[1] A. Damnjanovic, J. Montojo, Y. Wei, T. Ji, T. Luo, M. Vajapeyam, T. Yoo, O. Song, and D. Malladi, "A survey on 3GPP heterogeneous networks," IEEE Wireless Communications, vol. 18, no. 3, pp. 10-21, 2011.

[2] A. Ghosh, N. Mangalvedhe, R. Ratasuk, B. Mondal, M. Cudak, E. Visotsky, T. A. Thomas, J. G. Andrews, P. Xia, H. S. Joet al., "Heterogeneous cellular networks: From theory to practice," IEEE Communications Magazine, vol. 50, no. 6, pp. 54-64, 2012.

[3] H. Munir, S. A. Hassan, H. Parveiz, Q. Ni, "A Game Theoretical Network-Assisted User-Centric Design for Resource Allocation in 5G Heterogeneous Networks", IEEE Vehicular Tech. Conf. (VTC-Spring), May, 2016.

[4] E. Hossain, et al., "Evolution toward 5G multitier cellular wireless networks: An interference management perspective," in Wireless Commun. vol. 21, no. 3, pp. 118-127, 2014.

[5] J. Andrews, S. Singh, Q. Ye, X. Lin, and H. Dhillon, "An overview of load balancing in HetNets: Old myths and open problems," IEEE Transactions on Wireless Communications, vol. 21, no. 2, pp. 18-25, Apr. 2014

[6] Q. Ye, B. Rong, Y. Chen, M. Al-Shalash, C. Caramanis, and J. Andrews, "User association for load balancing in heterogeneous cellular networks," IEEE Transactions on Wireless Communications, vol. 12, no. 6, pp. 2706-2716, Jun. 2013.

[7] Y. Wang, S. Chen, H. Ji, and H. Zhang, "Load-aware dynamic biasing cell association in small cell networks," IEEE International Conference on Communications (ICC), pp. 2684-2689, Jun. 2014

[8] H. Klessig, M. Günzel, and G. Fettweis, "Increasing the capacity of large-scale HetNets through centralized dynamic data offloading," IEEE 80th Vehicular Technology Conference (VTC), Sep.2014.

[9] S. Rangan, T. S. Rappaport, and E. Erkip, "Millimeter-wave cellular wireless networks: Potentials and challenges", IEEE Proceedings, vol. 102, no. 3, pp. 366-385, 2014.

[10] X. Zhang and J. Andrews, "Downlink cellular network analysis with multi-slope path loss models," IEEE Trans. on Commun.,vol. 63, no. 5, pp. 1881-1894, May 2015.

[11] M. Ding, P. Wang, D. L'opez-P'erez, G. Mao, and Z. Lin, "Performance impact of los and nlos transmissions in small cell networks," IEEE Trans. Wireless Commun., Mar 2015.

[12] N. Garg, S. Singh, and J. Andrews, "Impact of dual slope path loss on user association in hetnets", IEEE Globecom Workshops, pp. 1-6, 2015.

[13] S. Sun et al., "Path loss, shadow fading, and line-of-sight probability models for 5G urban macro-cellular scenarios," IEEE Global Communications Conference, Exhibition \& Industry Forum (GLOBECOM) Workshop, Dec. 2015. 